\title{
Sputnik II como espectáculo: Arquitectura y cuerpo en el espacio
}

Sputnik II as spectacle: Architecture and body in space

\section{David Jiménez Moreno}

Universidad Politécnica de Madrid, España

david.anarqio@gmail.com

https://orcid.org/0000-0002-0586-7709 


\section{Resumen}

La primera forma de vida compleja en orbitar la Tierra lo hizo en un artefacto programado para asesinarla. A pesar del avance revolucionario que trajo el Sputnik II, detrás de sus apariencias futuristas persiste la misma ideología que busca entender el universo y relacionarse con él en términos de dominio y destrucción. La cápsula espacial no puede entenderse sin el usuario que alojaba, Laika, una perrita mestiza de tamaño mediano que sobrevivió en las calles de Moscú antes de ser recogida y sometida a un entrenamiento extremo. La rapidez de adaptación en los entrenamientos y su capacidad para la socialización con los humanos convertía a los canes en candidatos ideales con los que experimentar, pero los rusos contaban además con otro factor añadido, la herencia de los estudios realizados décadas antes por Ivan Pavlov desvelando detalladamente su fisiología y psicología. El marco en el que se ideó el Sputnik II fue uno de los momentos álgidos de la Guerra Fría, cuando las dos potencias vieron el espacio exterior como el próximo escenario en el que lucirse y derrotar a su adversario. La rivalidad entre superpotencias provocó que la creación y lanzamiento del Sputnik II fuera apresurado y sin motivaciones científicas que lo sustenten, fue concebido para el espectáculo.

\section{Palabras clave: Sputnik II; Laika; Guerra Fría; Carrera Espacial}

\section{Abstract}

The first complex life form to orbit Earth did so in an artefact programmed to kill it. Despite the revolutionary advance brought about by Sputnik II and behind its futuristic appearances lay the same persisting ideology that sought to understand the universe and relate to it in terms of domination and destruction. The space capsule cannot be understood without the user it housed, Laika, a medium-sized mixed-breed dog who survived Moscow's streets before being collected and subjected to extreme training. Dogs' speed of training adaptation and their ability to socialise with humans make them ideal experimentation candidates. Yet there was an added factor in the case of the Russians: the heritage of the studies conducted decades before by Ivan Pavlov, who detailed their physiology and psychology. The framework in which Sputnik II was devised was a high point in the Cold War, when the two superpowers saw in outer space a new scenario in which to show off and defeat their adversary. Such rivalry caused the creation and launch of Sputnik II to be rushed. The launch was not supported by scientific motivations, it was conceived for the show.

Key words: Sputnik II; Laika; Cold War; Space Race

Para citar este artículo / To cite this article:

MORENO, J.C Sputnik II como espectáculo: Arqui-tectura y cuerpo en el espacio. En: [i2] Investigación e Innovación en Arquitectura y Territorio [en línea]. 2022, Vol. 10, Núm. 1. ISSN: 2341-0515. https://doi.org/10.14198/I2.20284

Este trabajo se publica bajo una licencia de Creative Commons Reconocimiento 4.0 Internacional (CC BY 4.0): https://creativecommons.org/licenses/by/4.0/deed.es_ES 


\section{La potestad humana para experimentar con la otredad en los inicios de la exploración espacial}

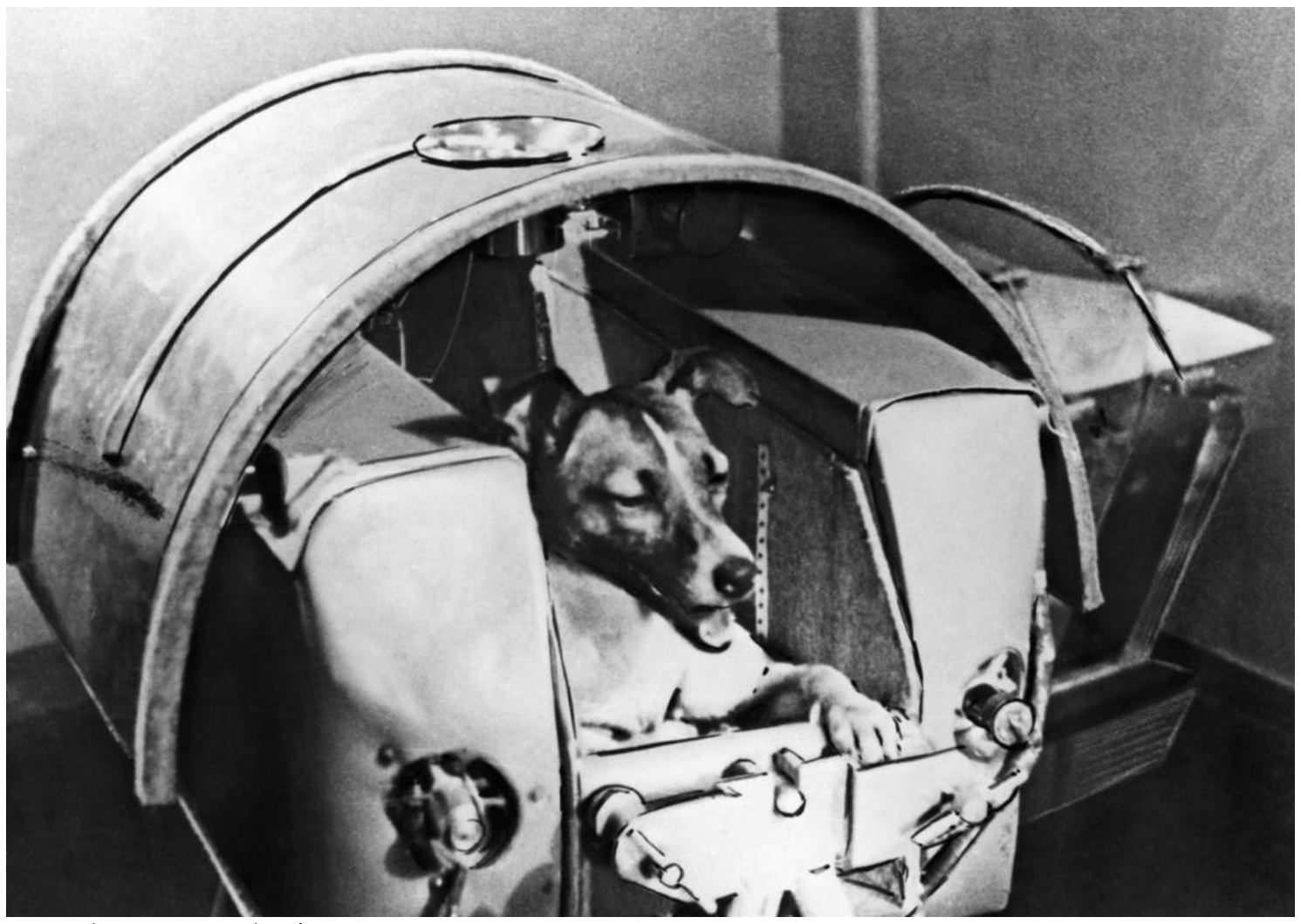

Fig. 1. Laika expuesta ante las cámaras.

La primera forma de vida compleja que orbitó la Tierra lo hizo en un artefacto programado para asesinarla. Kudryavka, rebautizada como Laika, era una perra mestiza de tamaño mediano que sobrevivía en las calles de Moscú. Los soviéticos habían desarrollado una amplia experiencia lanzando perros en cohetes hasta elevadas altitudes, pero no habían conseguido superar la barrera marcada por la atmósfera terrestre cabina (Nelson 2017). La rapidez de adaptación en los entrenamientos y su capacidad para la socialización con los humanos convertía a los canes en candidatos ideales con los que experimentar, pero los rusos contaban además con otro factor añadido, la herencia de los estudios realizados décadas antes por Ivan Pavlov desvelando detalladamente su fisiología y psicología cabina (Nelson 2017). Los animales seleccionados para las pruebas de vuelos orbitales debían ser hembras, debido a que su anatomía facilitaba la colocación del traje antigravedad y los instrumentos que debían almacenar sus desechos orgánicos. Solían recoger animales que vagaban por las calles, dando por hecho que estaban acostumbrados a soportar duras condiciones de hambre y frío, que ya estaban curtidos en la adversidad. Los perros seleccionados eran sometidos a entrenamientos extremos sin descanso durante días para que asimilaran las dificultades del lanzamiento. Les introducían en centrifugadoras que reproducían la velocidad del cohete, les sometían a ruidos exagerados similares a las explosiones y chirridos de la maquinaria, y les encerraban en espacios sucesivamente más pequeños para acostumbrarlos a las escuetas dimensiones de la cabina (Nelson 2017). En las durísimas pruebas destacó Laika, con solo tres años, pero mostrando una tranquilidad que sorprendió a los encargados del programa de adiestramiento (fig. 1). 
Las perras de los vuelos orbitales fueron modificadas en laboratorio para optimizar sus características biológicas a las necesidades concretas de los experimentos (Nelson 2017). Se les sometía a una cirugía para desplazar su arteria carótida hacia un pliegue de piel en la zona exterior del cuello, donde posteriormente se colocaba un dispositivo para medir la presión arterial que podía conectarse a un sistema para monitorizarla. Los encargados del programa espacial soviético ni si quiera se cuestionaron la legitimidad acerca de su capacidad para realizar las modificaciones físicas o los entrenamientos infernales a los que sometieron a los animales, dieron por hecho que podían, y lo hicieron. La facultad que la humanidad se ha concedido a sí misma para determinar el destino del resto de seres vivos ha tenido un impacto letal sobre los ecosistemas terrestres, perturbando el mundo entero por el privilegio que asume una sola

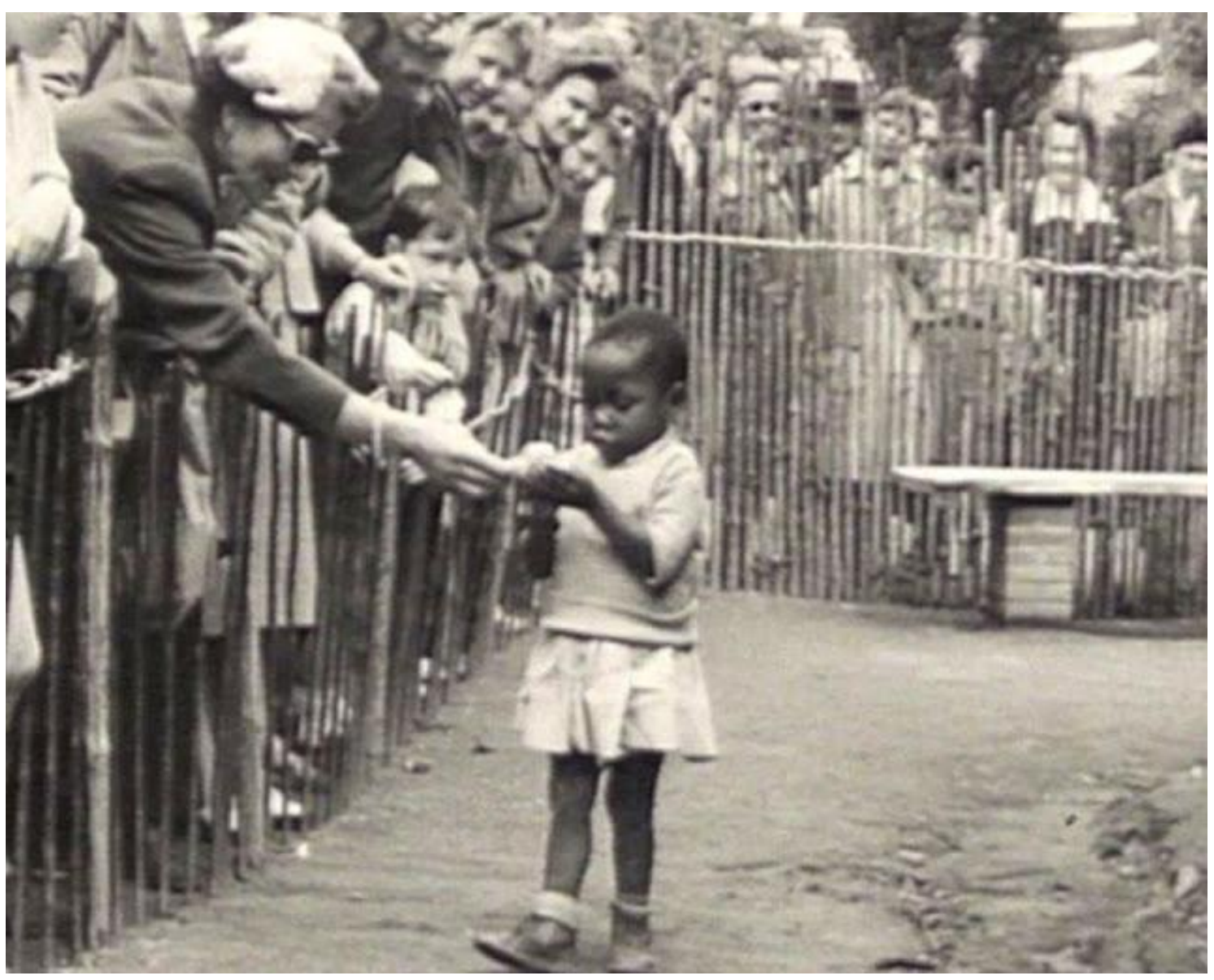

especie.

Fig. 2. Niña expuesta ante el público de Kongorama 
La atribución de derechos con pretensión de proteger únicamente a la humanidad tiene como consecuencia directa la exclusión del resto de seres vivos, consintiendo tácitamente el abuso sobre ellos. Braidotti afirma que en la definición de humanidad es tan relevante lo que incluye como lo que expulsa (Braidotti 2014), y este cisma determina de manera concluyente que los expulsados carecen de atributos propios que importen, que solo tenga valor la proyección de intereses que la humanidad establece sobre ellos. En palabras de Frazer, las personas "confunden el orden de sus ideas con el orden de la naturaleza y por ello imaginan que el dominio que ellos tienen o creen tener sobre sus pensamientos les permite ejercer el correspondiente dominio sobre las cosas.» (Frazer 1994).

La definición de humano siempre ha sido difusa y pocas veces se ha empleado con neutralidad. Un claro ejemplo de ello tuvo lugar pocos meses después del lanzamiento de Laika en otro evento con anhelos de ser una referencia global: la Exposición General Bruselas de 1958. El Ministerio de Colonias belga desarrolló una exhibición llamada Kongorama, un zoo humano (Fig. 2). ${ }^{1}$ Hicieron creer a un grupo de africanos provenientes de las posesiones coloniales belgas que viajarían a Europa para un intercambio cultural, pero una vez llegados a Bruselas se les condujo a la réplica de una aldea congoleña. Encerrados tras una cerca de bambú, debían deleitar a los espectadores con el exotismo que les atribuían, aguantando burlas y lanzamientos de plátanos, cacahuetes y monedas. La humanidad ha demostrado a lo largo de los siglos actuar de forma segmentaria, y de hacerlo "binariamente, según grandes oposiciones duales» (Deleuze y Guattari 2004). Esta postura impone una partición radical del universo que genera categorías antagónicas de manera automática. Si en un lado está lo humano, por correspondencia, en el otro, se sitúa lo no humano. Si a lo humano se le concede valor, a lo contrario no; si lo humano merece protección, lo contrario no. Esta frontera artificial e infranqueable cercena nuestro vínculo con el mundo y nos aísla de la vida al arrastrarnos a una concepción restringida de la misma. Skolimowski afirma que «la deficiencia de nuestro código para interpretar la naturaleza conduce a la deficiencia en nuestra relación con ella» (Skolimowski 2017). La deficiencia en la interpretación resulta en la deficiencia de la relación, y en estos términos, la concepción antropocentrista requiere un universo cerrado a la diversidad y la compasión, desarrollando una categoría dominante que debe atrofiar o asimilar aquello que toca, porque lo que no es humano está en el bando contrario. Es la anestesia del corazón y la justificación de la explotación.

\footnotetext{
1 Véase https://www.theguardian.com/world/2018/apr/16/belgium-comes-to-terms-with-human-zoos-of-itscolonial-past
} 


\section{La arquitectura como ejecutora de la ideología}

El contexto global en el que ambos eventos se desarrollaron define el trasfondo de su naturaleza. La exposición fue la primera feria mundial que tuvo lugar tras la Segunda Guerra Mundial, y se basó en el progreso técnico y científico originados en la era atómica. El tema de la exposición era: "evaluación del mundo para un mundo más humano», con lo que pretendía poner a la especie como paradigma y al mismo tiempo medida del progreso. Tan solo diez años después de que se aprobara la Declaración de Derechos Humanos, Europa acogía un zoo constituido por personas, lo que lleva a sospechar que a los africanos enjaulados no se les atribuía la condición de humanos. Sin embargo, el foco del debate sobre lo humano no estaba sobre ellos, sino en asuntos técnicos que centraban la gran rivalidad de la feria, la pugna entre la Unión Soviética y Estados Unidos para promover la supremacía de sus respectivos sistemas ante un mundo sumido en la Guerra Fría.

El marco en el que se ideó el Sputnik II fue uno de los momentos álgidos del conflicto, cuando las dos potencias vieron el espacio exterior como el siguiente escenario en el que exhibirse y derrotar a su adversario. Tras el lanzamiento del Sputnik I en octubre de 1957 el Primer Secretario del Partido Comunista, Nikita Jrushchov, ordenó la inmediata preparación de otro lanzamiento para el cuadragésimo aniversario de la Revolución Bolchevique. Con apenas un mes para alcanzar la fecha y sin posibilidad de negarse a la exigencia, el equipo de ingenieros al mando de Sergei Korolev rediseñó una copia del Sputnik I con una cápsula presurizada. El lanzamiento de Laika, precipitado y sin razones científicas que lo respaldaran, fue el equivalente a unos fuegos artificiales disfrazados de pulso tecnológico para la fiesta comunista. Un éxtasis que evidenciara la capacidad socialista de cambiar a la humanidad (Vulosevic 2020). Laika representaba a la perfección. El ideal de ciudadanía soviética, era dócil, de una raza mestiza que podía equipararse a una condición proletaria. Su lanzamiento a las estrellas era el alzamiento mismo del ideal comunista (Vulosevic 2020).

A pesar de las diferencias aparentes entre el Sputnik II y Kongorama, ambos espacios fueron concebidos para entretener y exhibir músculo. El diseño final del Sputnik II medía cuatro metros de altura por dos metros de base. Estaba compuesto por tres departamentos distintos. En la parte superior se introdujeron instrumentos científicos dedicados a tomar mediciones de rayos $X$ y radiación ultravioleta, así como células fotoeléctricas para registrar la radiación solar. Por debajo de ellos se encontraba un transmisor de radiofrecuencia muy similar al que se envió con el Sputnik I, encargado de emitir una señal constante que permitiera controlar la posición de la nave y los datos biológicos del animal. El nivel inferior del Sputnik II es el que debía albergar a Laika y garantizar su supervivencia, al menos durante el tiempo necesario para satisfacer los objetivos humanos. 
La cabina era una modificación de las que habían empleado previamente para lanzar perros en cohetes balísticos. Completamente hermética y construida con aluminio, tenía 80 centímetros de largo y 60 de diámetro, y estaba equipada con sensores para medir la respiración de Laika, así como su presión sanguínea y ritmo cardíaco. En lados opuestos de la cabina se colocaron unos paneles para renovar la atmósfera interior que contenían componentes alcalinos altamente activos para producir oxígeno y consumir el dióxido de carbono y el vapor de agua del interior. El cinturón de munición de una ametralladora se modificó para servir como dispensador de alimentos gelatinosos que se le entregaban a Laika en raciones periódicas, y este era además el instrumento que debía asesinarla en el momento preciso.

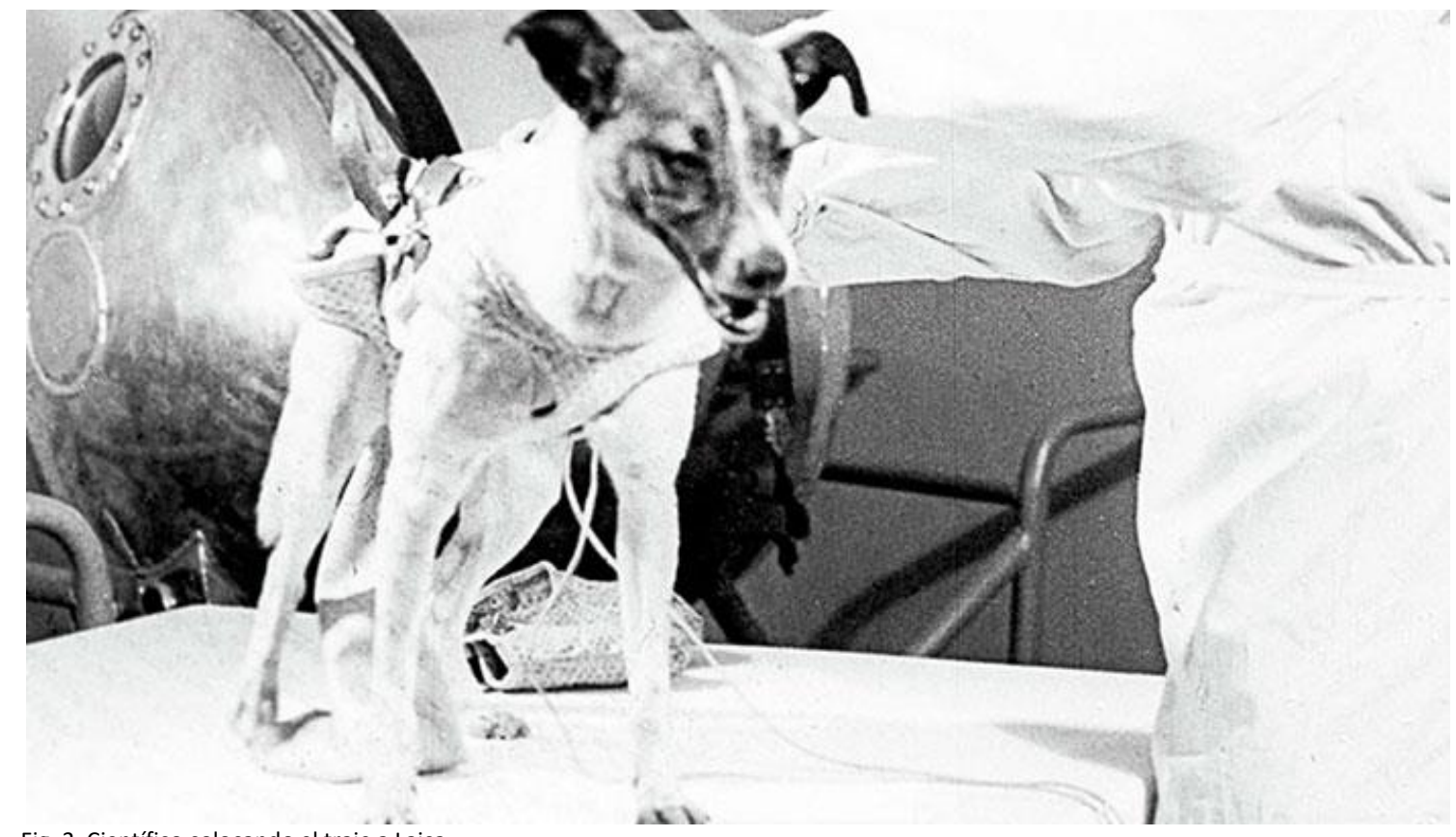

Fig. 3. Científico colocando el traje a Laica.

El traje espacial era el interfaz de conexión entre la biología de Laika y el Sputnik II (fig. 3) (Nelson 2017). Era la herramienta que recogía sus deposiciones y la que monitorizaba cada dato de la información que los humanos esperaban recoger. Estaba concebido para analizar su presión arterial, la frecuencia con la que latía su corazón, su respiración, sus ondas cerebrales, y para realizar electromiogramas para ver cómo funcionaba su sistema nervioso (Nelson 2017). El traje y el animal se fundían hasta el punto de que era difícil apreciar dónde empezaba uno y acababa el otro (Vujosevic 2020). La cabina acolchada tenía el tamaño justo para permitir que se tumbara o se pusiera de pie, los demás movimientos los tenía restringidos por un arnés que la mantendría encadenada, porque esa era la función que esperaban de Laika, mantenerse quieta mientras hacían lo que querían con ella. La primera vida lanzada al espacio exterior debía quedarse quieta en un espacio oscuro y asfixiante hasta que el cinturón de munición le dispensara la dosis letal como en un juego de ruleta rusa con el resultado final amañado. 
Kongorama ocupó casi ocho hectáreas divididas en siete pabellones que incluían temas relacionados con la agricultura, las misiones católicas o la minería en las posesiones coloniales belgas. Varios centenares de personas ataviadas con vestimentas africanas habitaban unas chozas de paja diminutas. Durante el día se les conminaba a realizar trabajos de artesanía y otras actividades que agradaran a los visitantes de la exposición (fig. 4). Kongorama se situó junto al Atomium, el edificio insignia de la exposición y símbolo de la actual Bruselas (fig. 5). La estructura mostraba un aspecto futurista cuando se erigió, contrastando claramente con el pabellón colonial, pero ambos se alineaban perfectamente con el tema de la feria. El progreso humano que se presentaba estaba basado en el dominio sobre la naturaleza, y a esa naturaleza pertenecían tanto el cristal de hierro aumentado del Atomium como las personas exhibidas en Kongorama. Lo que representaba a lo humano era el halo supremacista que atribuían a la civilización occidental, considerada como único sinónimo de progreso. Los seres que no estaban incluidos en ella quedaban fuera de la definición de humanidad, eran invisibles, y la invisibilidad, según Puig de la Bellacasa conlleva una evidente consecuencia ontológica (Puig de la Bellacasa 2014).
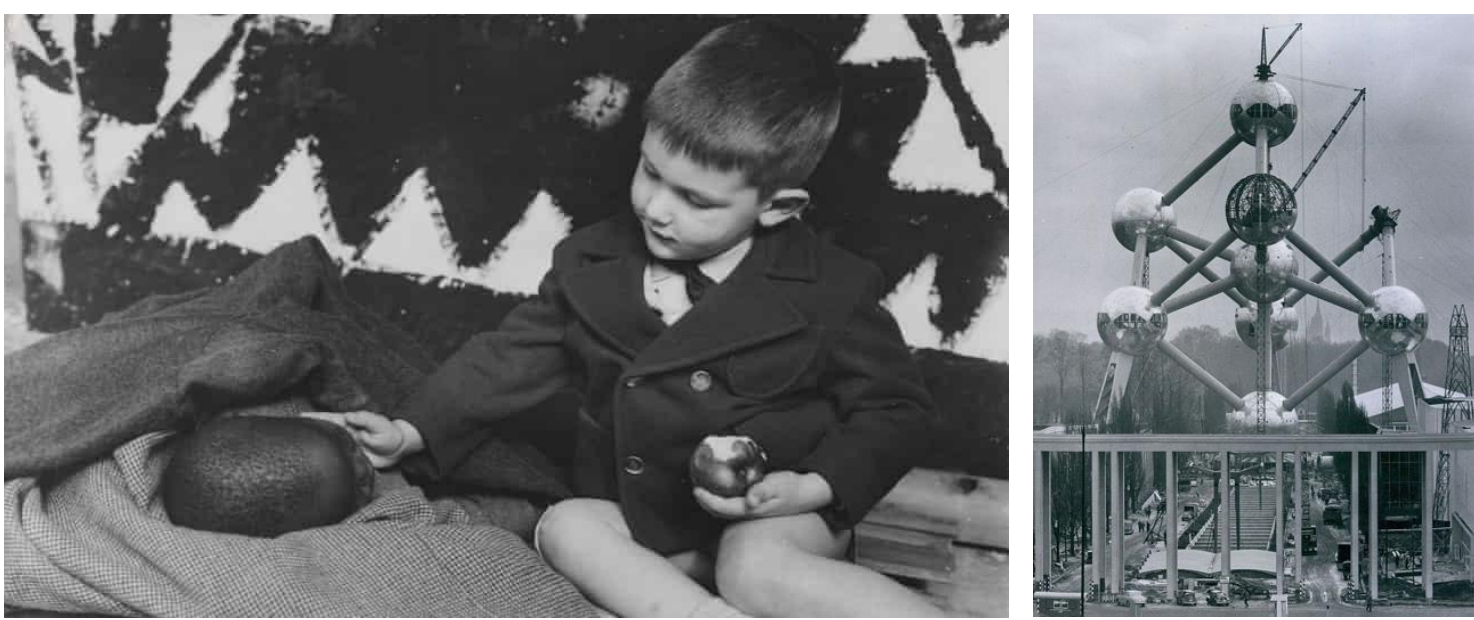

Fig. 4 lizda.) Niño acariciando a otro encerrado en Kongorama. Fig. 5. (dcha) Construcción del Atomium como emblema de la feria mundial de 1958. 
Tanto el Sputnik II como Kongorama fueron construidos como símbolos evidentes de progreso, pero las consecuencias nefastas contra la vida que los habitó obligan a cuestionar la dirección hacia la que se avanzó, o en favor de qué se avanzó a su costa. Lo que es evidente es que no hubo ningún adelanto para Laika ni para las personas encerradas en el zoo. El mito del progreso se ha erigido como un axioma incuestionable en Occidente, pero sin un objetivo claro al que avanzar, sostenido por una visión excluyente de la otredad, se convierte en un mero imperativo instrumental que exige ser eficiente y dominante para proceder a conquistas superficiales. La visión del progreso queda banalizada, reducida a un mero desarrollo de la tecnología, convertida en una búsqueda sin fin que desarraiga aún más a la humanidad del universo. El progreso de la técnica se impone al progreso de la vida, el medio se convierte en el fin, y sigue avanzado por el mero hecho de avanzar a costa de depredar un mundo sometido. En su juego de dualidades, la humanidad queda excluida de la categoría dominante y vuelve a formar parte de la naturaleza, cae a la categoría de lo sometido, cayendo en «la esclavitud de la misma tecnología que creamos para liberarnos», como afirma Harpur (Harpur 2013). 


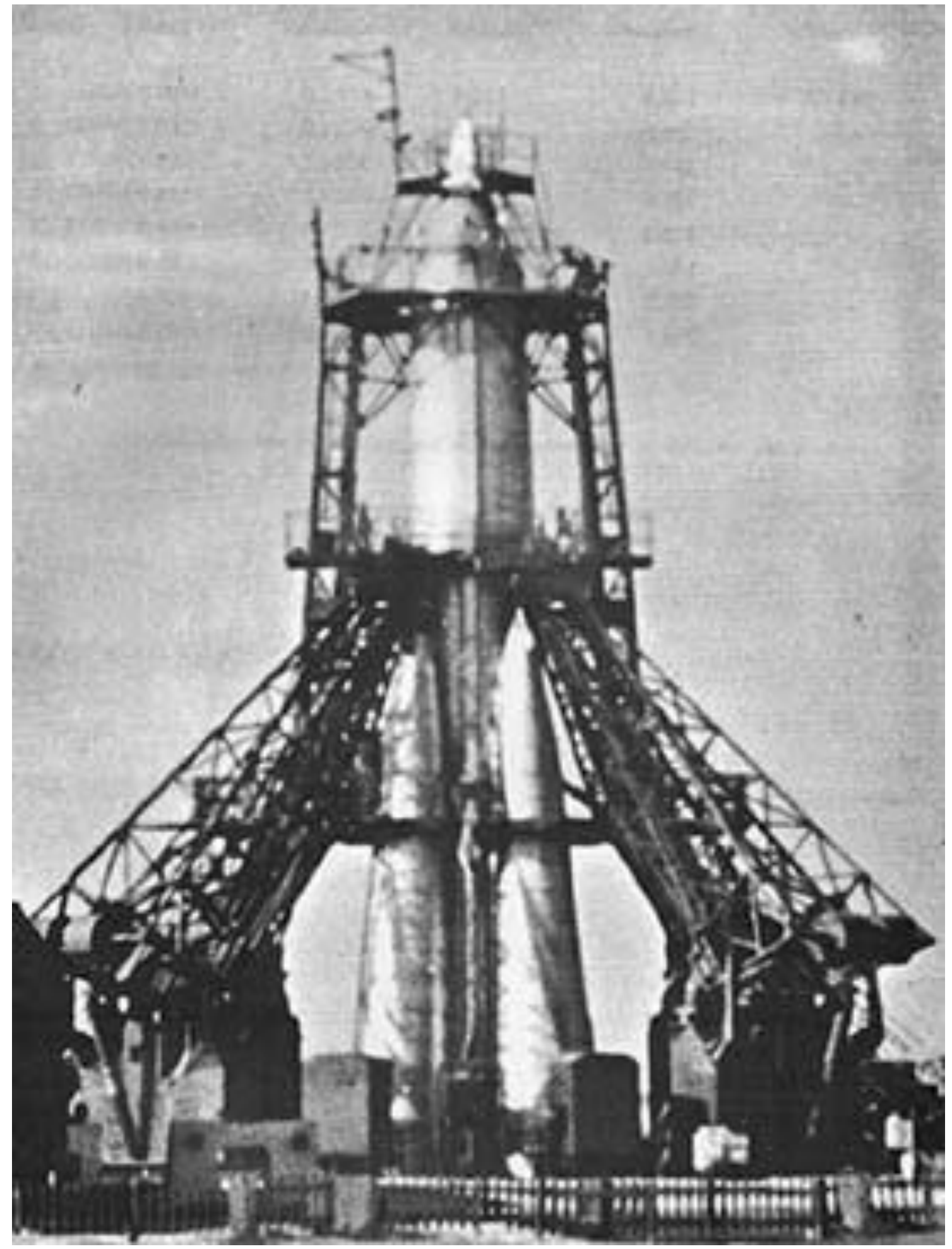

Fig 6. Cohete R7 empleado para lanzar los Sputniks. 


\section{Cambio de máscaras, mismo rostro}

El calvario final de Laika comenzó cuatro días antes del lanzamiento (fig. 6). Fue encerrada en la pequeña cápsula de la nave para que los ingenieros acabaran de pulir algunos aspectos con ella dentro, sabiendo que ya no saldría. ${ }^{2}$ Las bajas temperaturas en el cosmódromo de Baikonur hicieron necesaria una instalación para proporcionar calor al animal y evitar que tuviera complicaciones de salud antes de la misión. Al alcanzar la órbita planeada, la parte superior de la nave se desprendió exitosamente; sin embargo, la sección correspondiente al cohete no lo hizo, inhibiendo los instrumentos de control térmico, haciendo que la temperatura y la humedad de la cabina aumentaran drásticamente. Los primeros datos obtenidos de Laika indicaban que estaba aterrada. Su pulso estaba disparado, su respiración no dejaba de acelerarse desde que se inició el lanzamiento, y la temperatura de la cápsula aumentó hasta volverse insoportable. Los soviéticos mantuvieron durante décadas que Laika sobrevivió una semana, que su vida finalizó con una eutanasia, tal y como planearon los ingenieros. La verdad salió a la luz muchos años después, Laika sobrevivió entre cinco y siete horas. El miedo y el calor al que estuvo sometida en la cápsula acabaron con su vida. Los restos del Sputnik II continuaron orbitando durante cinco meses más antes de desintegrarse en la atmósfera.

Por primera vez, una criatura orbitaba la Tierra, pero el viaje estaba condenado desde el comienzo. El primer habitáculo de las estrellas estaba diseñado para acabar asesinando al pasajero que debía albergar. Se concibió para la muerte y nunca para vida porque la vida que albergaba nunca importó.

Oleg Gazenko, el científico encargado de entrenar a Laika para la misión, llegó a mostrarse arrepentido años más tarde, afirmando que ni siquiera se aprendió lo suficiente en la misión como para justificar una muerte semejante. El viaje de Laika fue una exhibición cuidosamente concebida para la propaganda, pero tras su apariencia falsamente futurista se escondía el sacrificio de otro animal para satisfacer a unos ídolos impertérritos. Si se deja a un lado la escenografía tecnológica no hay diferencia entre su muerte y cualquier otra realizada mil años atrás para satisfacer a los dioses y suplicarles que trajeran la lluvia o alejaran las plagas. EI contraste entre el Sputnik II y Kongorama es tan solo una ilusión, su propósito esencial era el mismo, el puro espectáculo en una pugna entre naciones.

Cada caso mostraba un mundo en etapas opuestas, uno colonial que se derrumbaba, otro extraterrestre que daba sus primeros pasos. Pero tras las apariencias persiste la misma ideología, una misma forma de entender el universo y relacionarse con él basada en la concepción de un grupo privilegiado con permiso a dominar y explotar aquello que queda fuera de su ámbito. La persistencia de esta corriente y los efectos destructivos que sigue generando hace que sea urgente plantear nuevas perspectivas que incluyan una comprensión más integral del universo y nuestra forma de relacionarnos con él. Para ello no es necesario negar la singularidad humana como defiende Haraway, (Haraway 1985) sino reconocer la importancia de lo que no es humano y establecer con ello una relación basada en la participación. Entender que lo que situado más allá de la frontera de lo humano no está ontológicamente separado de lo humano, como propone

\footnotetext{
2 Véase https://www.smithsonianmag.com/smithsonian-institution/sad-story-laika-space-dog-and-her-one-waytrip-orbit-1-180968728/
} 
Barad (Barad 2016). Concebir una forma de ser más transversal que se enlace de forma armoniosa con las realidades no humanas (Haraway 2019), un nuevo marco que permita desarrollar condiciones alternativas que promuevan valores capaces de acoger a la otredad.

La humanidad se encuentra en un punto de inflexión en su historia: la ascensión al universo, la salida del planeta natal. La relevancia del acto requiere una reflexión profunda. Ante nosotros se presenta la posibilidad de integrarnos en el universo y participar de él de manera íntima y plena, o la de exportar la explotación y el saqueo del entorno cósmico. En el resultado final de nuestra transformación como especie extraterrestre será decisiva la manera de concebir, percibir, y relacionarnos con el universo, porque ello determinará la forma en la que actuaremos en él.

Será fundamental la transformación que se lleve a cabo respecto al concepto de progreso, apartándolo de la noción abstracta y artificial actual, y conduciéndola hacia un avance significativo de la vida que permitan trascender dinámicas nocivas. Resolviendo el cisma entre la humanidad y el resto de forma que la integración de la humanidad en el universo sea la única forma de progresar en él. Una nueva relación establecida en términos de participación que nos reincorpore a la red transversal de relaciones que forma el espacio creado por la vida, capaz de reconciliar la falsa división entre sujeto y objeto, (Tranas 2016). humanidad y otredad, generando parentescos nuevos (Haraway 2019). A pesar de las décadas transcurridas desde el lanzamiento del Sputnik II y Kongorama, el afán de dominio de la humanidad ha suavizado sus formas, pero no ha variado su fondo en el preludio de la carrera espacial prevista para la próxima década. El asentamiento en lugares como la Luna se concibe cada vez más como una plataforma para esquilmar los recursos del satélite y enriquecer a unos pocos, para habitar Marte se plantea su terraformación, es decir, asimilar todo un planeta a las necesidades humanas, sin importar su naturaleza actual. Será necesario cambiar de perspectiva si no queremos exportar al cosmos la actitud devastadora con la que hemos arrasado nuestro propio planeta. 


\section{Referencias}

BARAD, K. Posthumanist Performativity: Toward an Understanding of How Matter Comes to Matter. Journal of Women in Culture and Society 2003, 28 (3), 2003, pp. 801-831. https://doi.org/10.1086/345321

BARAD, K. Meeting the Universe Halfway. En: Quantum Physics and the Entanglement of Matter and Meaning. 2007, Duke University Press. https://doi.org/10.2307/j.ctv12101zq

BARAD, K. No Small Matter Mushroom Clouds, Ecologies of Nothingness, and Strange Topologies of Spacetimemattering. Multitudes, 2016, 65(4). 64-74. https://doi.org/10.3917/mult.065.0064

BRAIDOTTI, R. Nomadic Ethics. Deleuze Studies, 2013, 7(3), 342-359. https://doi.org/10.3366/dls.2013.0116

BRAIDOTTI, R. Writing as a Nomadic Subject. Comparative Critical Studies, 2014, 11(2-3), $163-184$. https://doi.org/10.3366/ccs.2014.0122

BRAIDOTTI, R. The Critical Posthumanities; or, Is Medianatures to Naturecultures as Zoe Is to Bios? Cultural Politics, 2016 12(3), 380-390. https://doi.org/10.1215/17432197-3648930

BRAIDOTTI, R. Posthuman Critical Theory. Journal of Posthuman Studies, 2017, 1(1), 9-25. https://doi.org/10.5325/jpoststud.1.1.0009

BRAIDOTTI, R. A Theoretical Framework for the Critical Posthumanities. Theory, Culture \& Society, 2018, 1-31. https://doi.org/10.1177/0263276418771486

DELEUZE, G. y GUATTARI, F. Mil Mesetas: Capitalismo y Esquizofrenia. Valencia: Editorial Pre-Textos, 2004.

DOUGLAS KILGORE, D.W. Astrofuturism. En: Science, Race, and Visions of Utopia in Space. 2003, Philadelphia, University of Pennsylvania Press. https://doi.org/10.9783/9780812200669

FRAZER, J.G. La Rama Dorada. En: Magia y Religión, 1994, Ciudad de México, Fondo de Cultura Económica

HARAWAY, D. Manifesto for Cyborgs: Science, Technology, and Socialist Feminism in the 1980s. Socialist Review, $1985,70,65-108$.

HARAWAY, D. Simians, Cyborgs, and Women. En: The Reinvention of Nature. 1991, Nueva York, Routledge.

HARAWAY, D. When Species Meet. 2008, Mineápolis, University of Minnesota Press.

HARAWAY, D.J. Seguir con el problema. En: Generar parentesco en el Chtuluceno, 2019, Bilbao, Consonni.

HARPUR, P. El fuego secreto de los filósofos. 2013, Girona, Ediciones Atalanta.

HILLMAN, J. El pensamiento del corazón. 2017, Girona, Ediciones Atalanta.

LATOUR, B. We Have Never Been Modern. 1993, Cambridge, Harvard University Press.

LATOUR, B. Reassembling the Social: An Introduction to Actor-Network-Theory. 2007, Nueva York, Oxford University Press.

NELSON, A. What the dogs did: animal agency in the Soviet manned space flight programme En: BJHS Themes, 2017, 2, 79-99. https://doi.org/10.1017/bjt.2017.9

PUIG DE LA BELLACASA, M. Encountering bio-infrastructure. En: Ecological struggles and the sciences of soil. Social Epistemology, 2014, 28(1), 26-40. https://doi.org/10.1080/02691728.2013.862879

PUIG DE LA BELLACASA, M. Ecological thinking, materialist spirituality and the poetics of infrastructure. 2016, MIT Press, 47-68

PUIG DE LA BELLACASA, M. Matters of Care. Speculative Ethics in More Than Human Worlds. 2017, Minneapolis, University of Minnesota Press. https://doi.org/10.1017/S2753906700002096

SKOLIMOWSKI, H. Filosofía Viva En: La ecofilosofía como un árbol de la vida. 2017, Girona, España: Ediciones Atalanta.

TARNAS, R. La Pasión de la Mente Occidental. 2016, Girona, España: Ediciones Atalanta. 
VUJOSEVIC, Homage to the Sad Space Bitch. Laika-Belka-Strelka. En: Thresholds, 2020, (48), pp.148-157. https://doi.org/10.1162/thld_a_00718

\section{Bio}

Arquitecto y escritor, con dos novelas publicadas. Actualmente estoy en mi último año de doctorado en la Universidad Politécnica de Madrid, trabajo como investigador en el Grupo de Poetic Justice del MIT Media Lab, y también soy investigador visitante en la Agencia Espacial Europea, concretamente en su Centro Europeo de Astronautas.

\section{Bio}

Architect, with a bachelor's degree and a master's degree, and also a writer, with two published novels. I'm currently in my last year of PhD at the Polytechnic University of Madrid, I work as a researcher for the Poetic Justice Group of the MIT Media Lab, and I'm also a visiting researcher at the European Space Agency, specifically in its European Astronaut Center. 